International Journal of Engineering \&Technology, $7(3.12)(2018) 1213-1217$
International Journal of Engineering \& Technology
SPC
Website: www.sciencepubco.com/index.php/IJET
Research paper

\title{
Novel Approach of Neural Collaborative Filter by Pairwise Objective Function with Matrix Factorization
}

\author{
Ram Sethuraman $^{1^{*}}$, Akshay havalgi ${ }^{2}$ \\ *Corresponding Author E-mail: ${ }^{1}$ Ramsethuraman123@gmail.com, ${ }^{2}$ akshayh380@gmail.com
}

\begin{abstract}
The concept of deep learning is used in the various fields like text, speech and vision. The proposed work deep neural network is used for recommender system. In this work pair wise objective function is used for emphasis of non-linearity and latent features. The GMF (Gaussian matrix factorization) and MLP techniques are used in this work. The proposed framework is named as NCF which is basically neural network based collaborative filtering. The NCF gives the latent features by reducing the non-linearity and generalizing the matrix. In the proposed work combination of pair-wise and point wise objective function is used and tune by using the concept of cross entropy with Adam optimization. This optimization approach optimizes the gradient descent function. The work is done on $1 \mathrm{~K}$ and $1 \mathrm{M}$ movies lens dataset and it is compared with deep matrix factorization (DMF).
\end{abstract}

Keywords: NCF, Gaussian, movielens

\section{Introduction}

Now it is the time when we have a large collection of useful information related to the products and services on the internet. Recommender system plays an important role in the websites and online services related to the e-commerce and video streaming. Recommendation system used to provides most appropriate product or services to the user related to their interest and choices It is basically works as information filtering system on the basis of ranking and rating given by the users to the product on the website [1]. Recommendation is mainly based on the feedback and interest of users.
It is also an effective tool for personalization because it suggests the best thing according to the taste. This process is known as collaborative filtering. CF mainly predicts the interest of a particular user on the basis of similarity with past products selection. Rating is one of the effective methods used in collaborative filtering to extract the information from them [2].This approach is also differentiated into two types that are Memory-Based and Model Based. Matrix factorization is a technique of collaborative filtering approach which learns a latent space to represent a user or an item becomes a standard model for recommendation due to its flexibility and reliability. Latent space is basically predicts the personalized ranking over a set of items for each individual user with the similarity among the users and the products[3].

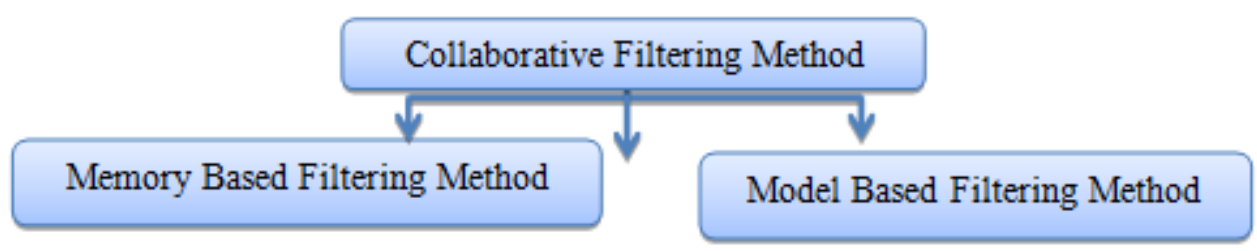

Fig. 1.1: Types of Collaborative filtering approach

Memory based: It uses the complete user item data to generate the prediction. It mainly uses statistical methods for filtering.

Model based: In the method firstly defined the user model by using the clustering, association, Bayesian and neural network. These methods are used to predict user's rating of unrated products.

In the proposed work collaborative filtering method is used in which two factor are considered that are user and item. On the basis of this work, some existing approaches are also considered to go through to enhance the knowledge about the collaborative filtering and its scope.

\section{Related work}

A recommender system based on collaborative filtering using ontology and dimensionality reduction techniques are proposed and worked on the issue of sparsity and scalability. Singular value decomposition approach finds the similar items and users in each cluster. Ontology is also worked well with the context based recommender system $[4,5]$. Collaborative filtering approach is some time faces the issue of cold start when the ratings of the product are not available or zero. When very few amounts of ratings are available for the new product and it is called as Incomplete Cold Start problem. This problem is resolved by using Time- Aware collaborative filtering with deep learning [6]. This 
type of problem is also solved by using the optimization approaches and algorithms. K-mean clustering with Cuckoo search Optimization algorithm is used in movie recommendation system on Movielens dataset. This system classifies the users on the basis of similarity in choice. Recommendation of this system is based on the $\mathrm{K}$-mean clustering and Cuckoo optimization algorithm [7].

As the technology changes day by day, a large number of techniques are employed in the field of recommendation system. In recent works deep learning has employed for effective recommendation. In this model the interaction between the user and item features is resorted to matrix factorization. Then this is applied to latent features of users and items. This approach is called as Neural Network-based Collaborative filtering [8]. A hybrid recommendation system by using ontology and neurofuzzy techniques is proposed movie recommendation to the users. Semantic approach is used to compute the similarity between the products and demographic similarity measure is applied between each pair of users. Fuzzy rules are applied to achieve the more accuracy in recommendation [9]. Accuracy of the system is also improved by using clustering and association rules mining on the implicit data. Clustering approach resolve the issue of sparsity in the huge amount of data. It also reduces the data size and dimensionality of the item space.Collaborative filtering gives more effective results when it is extended with other techniques even when sparsity is also present in the data[10].

Semantic-based friend recommendation system is proposed which suggest the friends on the basis of lifestyles. In this method similarity between the lifestyles are matched and then recommend the fiend on that similarity. Latent Dirichlet Allocation algorithm is used to extract the style of the users of Friend book. Similarity metrics are used to measure the similarity between the styles and impact. The proposed method is implemented on the android phones and performance is evaluated on small scale and large scale [11]. Rank Aggregation approach is used for the product recommendation. used the feedback of the users related to the product and score given by them to the related product. This feedback is used by the rank aggregation algorithm for the recommendation. The performance of this system is evaluated by using the parameter false negative rates, false positive rates and precision. The variation in result over the existing method shows the effectiveness of the proposed method [12].

\section{Proposed Approach Architecture}

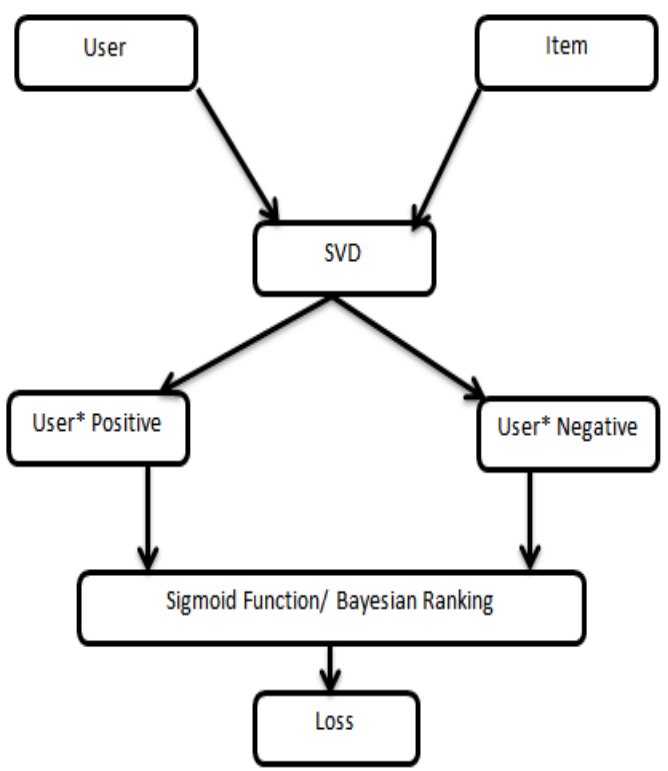

Fig. 3.1

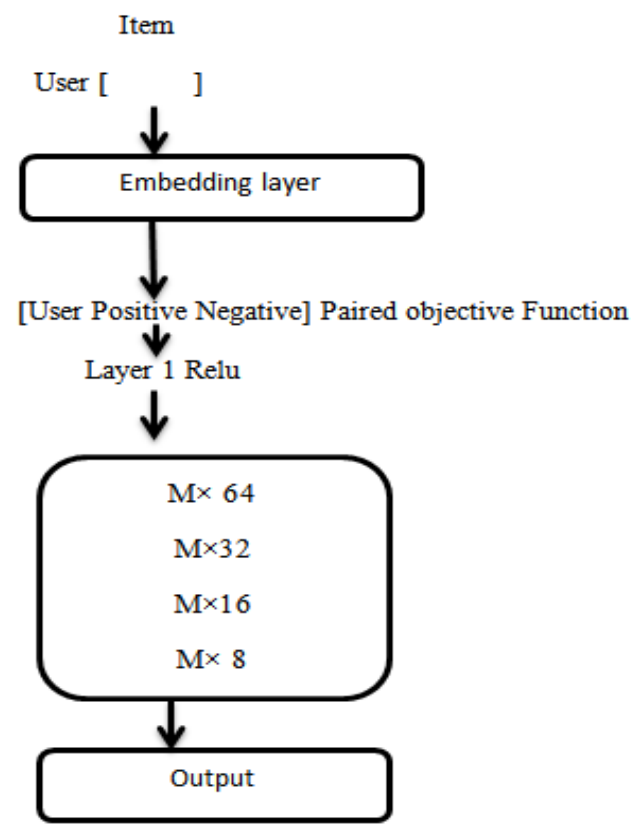

Fig. 3.2

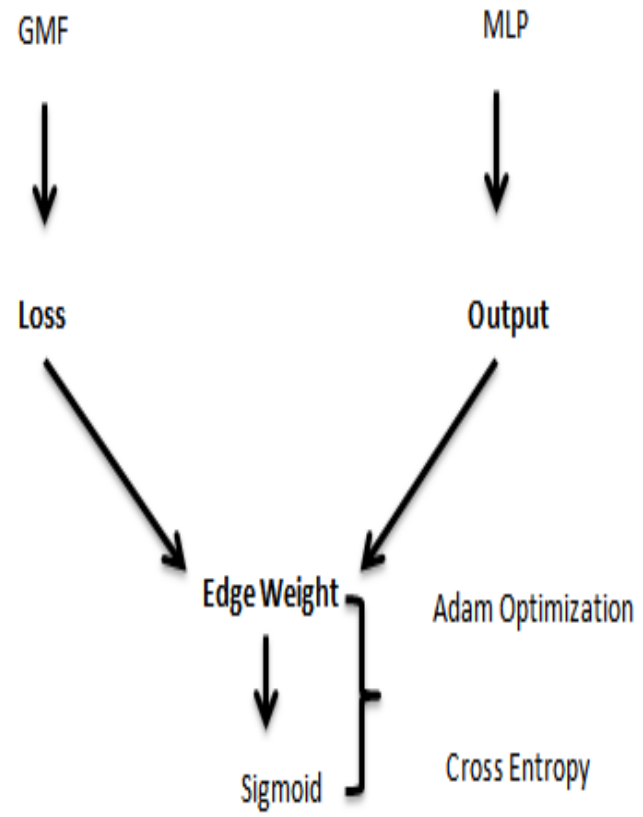

Fig. 3.3

In the above given figure 3.1 it represents the initial part of the proposed approach in which latent features of the User and Movie review matrix is extracted. After this SVD is applied on this matrix for matrix factorization and then pair-wise objective function by using Bayesian ranking approach. In Pairwise objective function it uses user matrix and movie review matrix which use implicit review $\{1,0\}$. This layer also calculate the los but it tune in the loss figure 3.1

In figure 3.2 it describes the MLP layer which extracts the threshold output by multilayer approaches. In this approach window size used is $(64,32,16$, and 8$)$. These layers also reduce the non-linearity by using Relu layer. From figure 3.1 get loss and figure 3.2 get output tune and figure 3.3 architecture by using loss function which tune by Adam optimization. By these architecture the main objective is completed by reduction in loss and reduce the nonlinearity by pairwise objective function. 


\section{Proposed Algorithm}

Step1 Fusion of Twomodels GMF (generalized matrix Factorization) and MLP (Multi-LayerPerceptron)

Sep2 Model based on implicit feedback

a. Input User * items

b. $\quad$ Latent vectors $\leftarrow$ Apply SVD on the rating matrix $\mathrm{R}$

c. $\quad \mathrm{GMF}=\mathrm{User}_{4 * 2} * \mathrm{Item}_{5 * 2}$ (Dot Product of the latent vectors)

d. $\quad M L P$

i. Assume $X$ number of hidden layers.

ii. Layer 1,

1. first, concatenate vertically the $\mathrm{p}_{\mathrm{i}}$ and $\mathrm{q}_{\mathrm{j}}$ latent vectors,

a. $\quad \mathrm{z} 1=[\mathrm{pi}, \mathrm{qj}]$

b. $\quad$ Output of layer 1 (to be fed into layer 2$)=>\mathrm{a} 1 *(\mathrm{~W} 1 * \mathrm{z} 1+\mathrm{b} 1) \ldots$

2. $\quad$ a1 $=$ activation function (sigmoid.)

$\mathrm{W} 1=$ Weights for instances, $\mathrm{b} 1=$ biasLayer $\mathrm{X}=$ The last layer

3. Output of the last layer $=\mathrm{a}_{\mathrm{n}} *\left(\mathrm{w}_{\mathrm{n}} * \mathrm{z}_{\mathrm{n}}+\mathrm{b}_{\mathrm{n}}\right)$

4. Concatenate outputs of GMF and MLP (vertical concatenation),

$\mathrm{OP}=\left[\quad\left[\mathrm{User}_{4} * 2 *\right.\right.$ Item $\left._{5 * 2}\right]$

$$
\left[\mathrm{a}_{\mathrm{n}} *\left(\mathrm{w}_{\mathrm{n}} * \mathrm{z}_{\mathrm{n}}+\mathrm{b}_{\mathrm{n}}\right)\right] \quad \text { ]........... }
$$

$\mathbf{Y i j}^{\prime}=\boldsymbol{\sigma} *(\mathbf{h} * \mathbf{O P})$

a. where, $\sigma=$ activation function,

b. $\quad h=$ edge weights of the $O / P$ layer

e. Minimize the "loss" function to tune the parameters

i. $\quad 1 / 2 \sum\left(\mathrm{Y}_{\mathrm{ij}}{ }^{\prime}-\mathrm{Y}_{\mathrm{ij}}\right)^{2}+(\lambda / 2)\left((\bmod (\mathrm{U}))^{2}+(\bmod (\mathrm{V}))^{2}\right)$

iii. $\quad$ Put the value of $\mathrm{Y}_{\mathrm{ij}}$ ' from equation (..1) above.

\section{Proposed Approach Description}

\section{Implicit Data}

Let $\mathrm{M}$ and $\mathrm{N}$ denote the number of users and items respectively so, matrix form

$$
Z \in R^{M \times N}
$$

$Z_{u, 1}=\left\{\begin{array}{cc}1, & \text { if user } U \text { given review for } \\ 0, & \text { otherwise }\end{array}\right.$

$R \leftarrow$ Vector Space

\section{Matrix Factorization (SVD)}

In SVD Matrix factorize in three matrixes.

In proposed approach

$A=$ User $_{m \times n} *$ Item $_{n \times m}$

$A=U S V^{T}$

$\mathrm{U} \leftarrow$ User Matrix

$V^{T} \leftarrow$ Item Matrix

$S \leftarrow$ Ranking Item

Apply MLP (Multi-Layer Perceptron)

$Z_{1}=\left[P_{i} Z_{i}\right]$

Latent Vector of Users and items

\section{Output Layer}

$O_{1}=a_{1} *\left(W_{1} * Z_{1}+b_{1}\right)$

$O_{2}=a_{2} *\left(W_{2} * Z_{2}+b_{2}\right)$ 
$O_{n}=a_{n} *\left(W_{n} * Z_{n}+b_{n}\right)$

$O_{n} \leftarrow$ last output of MLP Layer

\section{In MLP Use $Q_{1}$ (Sigmoid Function)}

$f(x)=\frac{1}{1+C^{-X}}$

\section{Objective Function}

In proposed approach pair wise objective function is used.

$\theta^{t+1}=\theta^{t}+\eta * \frac{\partial 0(\theta)}{\partial \theta}$

$\theta^{t+1} \leftarrow$ ranking of item at $(t+1)$ time

$\theta^{t} \leftarrow$ ranking of item at $t$ time

$\eta \leftarrow$ Learning rate

$\frac{\partial 0(\theta)}{\partial \theta} \leftarrow$ Minimizing Error

In pairwise objective function apply on the both latent and feature vector of user and item.

\section{Concatenate GMF and MLP}

In proposed use vertical concatenation of latent feature come from GMF and output come from MLP so,

$O P=\left[[\right.$ User $*$ Item $\left.] a_{n}\left(W_{n} * Z_{n}+b_{n}\right)\right]$

$Y_{i, j}^{\prime}=\sigma *(h * \partial P)$

$\sigma=$ activation Function

$h=$ edge weight od output layer

\section{Loss Function}

Tune the equation by loss function

Loss function $=1 / 2 \sum\left(Y_{i j}^{\prime}-Y_{i j}\right)^{2}+1 / 2\left(\left(\bmod (v)^{2}\right)+\left(\bmod (v)^{2}\right)\right) \ldots .$.

$Y_{i j}^{\prime}$ from equation (5.11)

\section{Results and Discussion}

\subsection{Experimental Settings}

Movie Lens: For collaborative filtering approach for movie rating dataset Movie lens is widely used. In the proposed work 1 Million ratings data set is used in which each user has at least 20 ratings. In this work implicit feedback is used for investigate the performance of the learning. On implicit data each entry is marked as 0 and 1 whether the user has rated the item.In this section, we compare the proposed NMF with DMF. As our proposed methods

aim to model the relationship between users and items, we mainly compare with user-item models.

Table 6.1: DMF (Deep Matrix Factorization)

\begin{tabular}{|c|c|c|}
\hline Dataset & NDCG & H.R \\
\hline Movie lens $100 \mathrm{~K}$ & 0.389 & 0.652 \\
\hline Movie Lens 1M & 0.381 & 0.623 \\
\hline
\end{tabular}

Observation 1: In the above given table 6.1 Comparison of DMF approach on the movie lens dataset with $100 \mathrm{~K}$ and 1Million instance is done. In analysis use NDCG which show that how much generalize matrix.In case of $100 \mathrm{~K} 38.9 \%$ and $1 \mathrm{M}$ show $38.1 \%$.In case of hit ratio (HR) it also decreases which is indirectly represent accuracy.

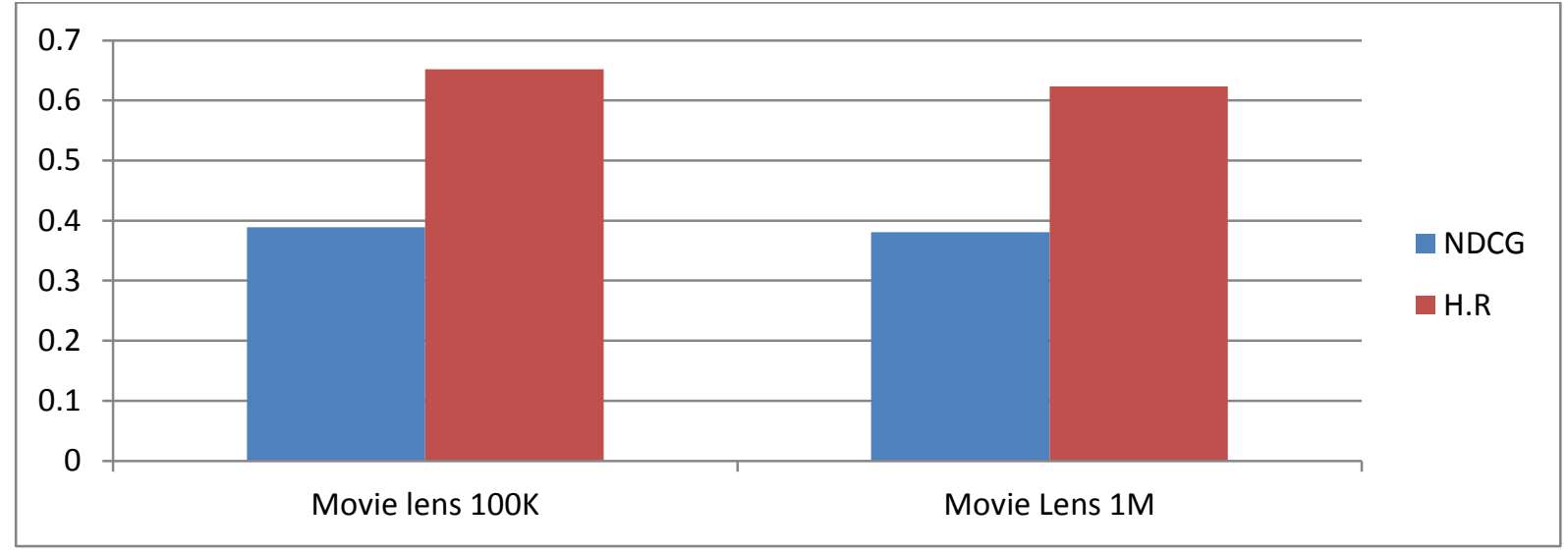

Fig. 6.1: Comparison between $1 \mathrm{~K}$ and $1 \mathrm{M}$ dataset with $\mathrm{DMF}$ 
Table 6.2: Proposed Approach Results

\begin{tabular}{|c|c|c|}
\hline Dataset & NDCG & H.R \\
\hline Movie lens 100K & 0.425 & 0.789 \\
\hline Movie Lens 1M & 0.415 & 0.892 \\
\hline
\end{tabular}

Observation2: In table 6.2the Comparison of proposed approach on the movie lens dataset with $100 \mathrm{~K}$ and 1Million instance is presented in the graphical form. In analysis NDCGis used which show that how much matrix is generalize. In case of $100 \mathrm{~K} 42.5 \%$ and $1 \mathrm{M}$ it is $41.5 \%$.In case of hit ratio (HR) it increase and show opposite behavior to DMF which indirectly represent the accuracy. The reason behind its improvement is pairwise objective function which generalize user and movie review at same time.

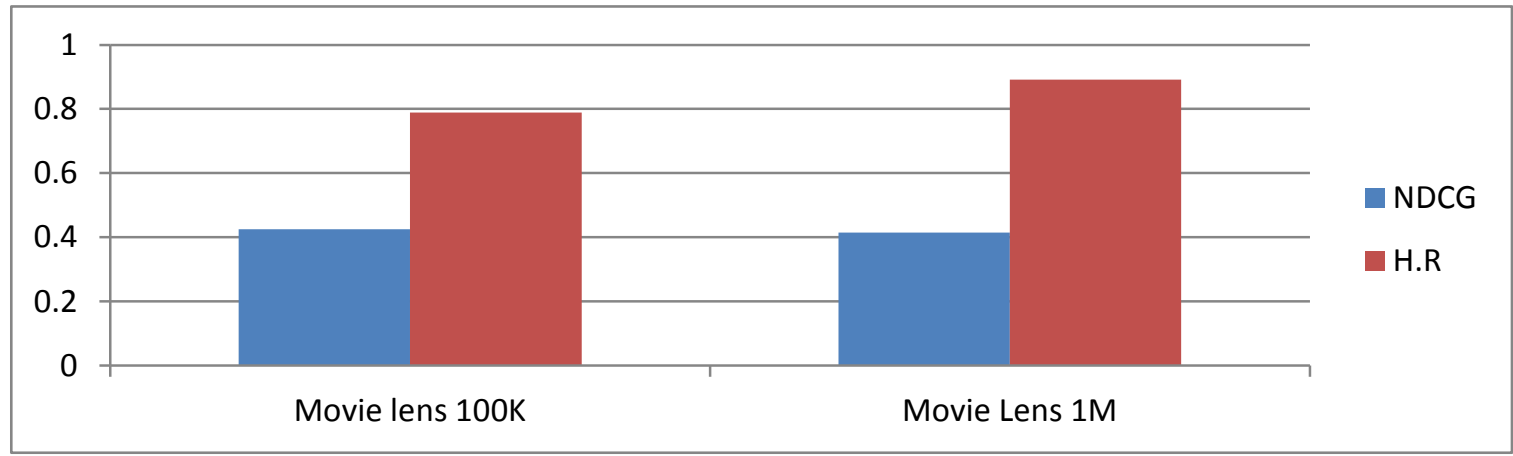

Fig. 6.2: Comparison between $1 \mathrm{~K}$ and $1 \mathrm{M}$ dataset with Proposed

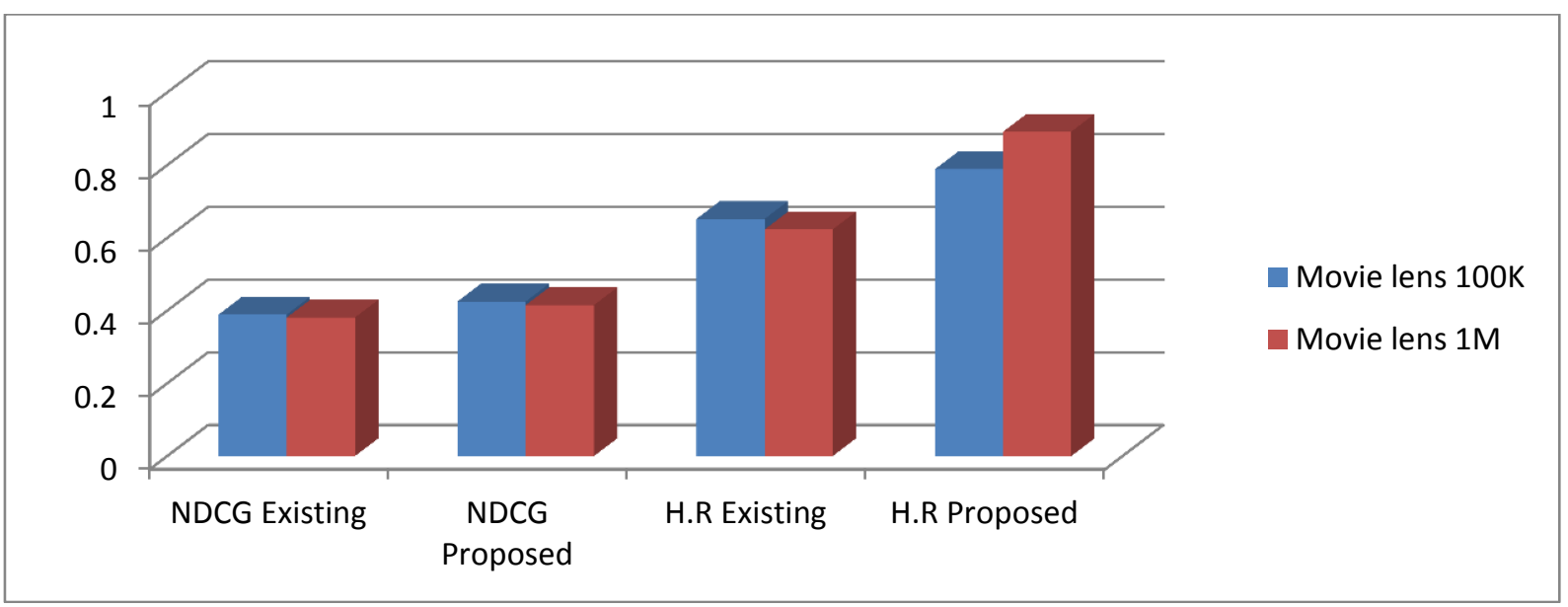

Fig. 6.3: Comparison of Proposed and Existing approach result

\section{Conclusion}

The proposed work explores the neural network architecture for collaborative filtering. The implicit movie reviews are generalized by NCF using Gaussian ranking and tune by loss function which is given in equation 5.11. The observations in the results clearly show the significant improvement in H.R. In this work pair wise objective function is used for emphasis of non-linearity and latent features and the proposed approach is combination of pairwise objective function with point wise objective and tune by cross entropy with Adam optimization which optimize version of gradient descent. In empirical analysis use $1 \mathrm{~K}$ and $1 \mathrm{M}$ movie lens dataset and compare with deep matrix factorization (DMF)

\section{References}

[1] Shah, Mit, Dhruvesh Parikh, and Bharat Deshpande. "Movie Recommendation System Employing Latent Graph Features in Extremely Randomized Trees." Proceedings of the Second International Conference on Information and Communication Technology for Competitive Strategies.ACM, 2016.

[2] Schnabel, Tobias, et al. "Using shortlists to support decision making and improve recommender system performance." Proceedings of the 25th International Conference on World Wide Web.International World Wide Web Conferences Steering Committee, 2016.

[3] Singh, Gurpreet, and Rajdavinder Singh Boparai. "A Novel Hybrid Music Recommendation System using K-Means Clustering and PLSA." Indian Journal of Science and Technology 9.28 (2016).
[4] Nilashi, Mehrbakhsh, Othman Ibrahim, and KaramollahBagherifard. "A recommender system based on collaborative filtering using ontology and dimensionality reduction techniques." Expert Systems with Applications 92 (2018): 507-520.

[5] Sulthana, A. Razia, and SubburajRamasamy. "Ontology and context based recommendation system using Neuro-Fuzzy Classification." Computers \& Electrical Engineering (2018).

[6] Wei, Jian, et al. "Collaborative filtering and deep learning based recommendation system for cold start items." Expert Systems with Applications 69 (2017): 29-39.

[7] Katarya, Rahul, and Om PrakashVerma. "An effective collaborative movie recommender system with cuckoo search." Egyptian Informatics Journal 18.2 (2017): 105-112.

[8] He, Xiangnan, et al. "Neural collaborative filtering." Proceedings of the 26th International Conference on World Wide Web.International World Wide Web Conferences Steering Committee, 2017.

[9] Kermany, NaimeRanjbar, and Sasan H. Alizadeh. "A hybrid multicriteria recommender system using ontology and neuro-fuzzy techniques." Electronic Commerce Research and Applications 21 (2017): 50-64.

[10] Najafabadi, Maryam Khanian, et al. "Improving the accuracy of collaborative filtering recommendations using clustering and association rules mining on implicit data." Computers in Human Behavior 67 (2017): 113-128.

[11] Wang, Zhibo, et al. "Friendbook: a semantic-based friend recommendation system for social networks." IEEE Transactions on Mobile Computing 14.3 (2015): 538-551.

[12] Sohail, ShahabSaquib, JamshedSiddiqui, and Rashid Ali. "User feedback based evaluation of a product recommendation system using rank aggregation method." Advances in Intelligent Informatics.Springer, Cham, 2015.349-358. 